SELECCIONES MATEMÁTICAS
Universidad Nacional de Trujillo
ISSN: $2411-1783$ (Online)
2021; Vol. 8(2): 326-332.

\title{
A proof of the Cayley-Hamilton theorem using algebraic geometry
}

\section{Una prueba del teorema de Cayley-Hamilton utilizando geometría algebraica}

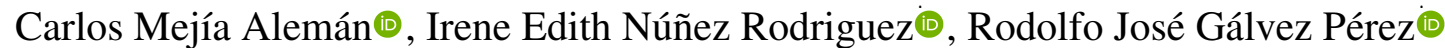 \\ and Neisser Pino Romero
}

Received, Oct. 20, 2021

Accepted, Dec. 10, 2021

How to cite this article:

Megía C, Núñez I, Gálvez R, Pino N. A proof of the Cayley-Hamilton theorem using algebraic geometry. Selecciones Matemáticas. 2021;8(2):326-332. http://dx.doi.org/10.17268/sel . mat.2021.02.09

\begin{abstract}
In this work, we will prove the Cayley-Hamilton theorem using algebraic geometry. We will see a different proof than the one seen in a linear algebra course, in this case we will use the Zariski topology, then we will take advantage of the fact that every square matrix of order $n \times n$, with entries in a field $\mathbb{K}$, denoted by $\left(a_{i j}\right)_{n \times n}$ can be seen as an element of the affine space of dimension $n \times n$ over the field $\mathbb{K}$ and thanks to this, we can resort to algebraic sets and algebraic varieties in order to obtain some results seen in an algebraic geometry and to get a proof of the Cayley-Hamilton theorem.
\end{abstract}

Keywords . Affine space, algebraic set, algebraic manifold, Zariski topology.

\section{Resumen}

En este trabajo, probaremos el teorema de Cayley-Hamilton utilizando geometría algebraica. Veremos una prueba diferente a la que se ve en un curso de álgebra lineal, en este caso utilizaremos la topología de Zariski, luego nos aprovecharemos de que toda matriz cuadrada de orden $n \times n$, con entradas en un cuerpo $\mathbb{K}$, denotada por $\left(a_{i j}\right)_{n \times n}$ puede ser vista como un elemento del espacio afín de dimensión $n \times n$ sobre el cuerpo $\mathbb{K}$ y gracias a esto podemos recurrir a los conjuntos algebraicos y a las variedades algebraicas para así obtener algunos resultados vistos en un curso de geometría algebraica y conseguir una prueba del teorema de Cayley-Hamilton.

Palabras clave. Espacio afín, conjunto algebraico, variedad algebraica, topología de Zariski.

1. Introducción. Este artículo está inspirado por el trabajo que publicaron Jeffrey A. Rosoff y Gustavus Adolphus College, que tiene por nombre A topological proof of the Cayley-Hamilton theorem, ver [8].

En este artículo veremos algunas propiedades de la geometría algebraica clásica. Como primera definición veremos lo que es un espacio afín sobre un cuerpo $\mathbb{K}$, luego veremos lo que es un conjunto algebraico y enunciaremos algunas propiedades y ejemplos relacionadas con dicho conjunto. Veremos quienes son los conjuntos cerrados de la topología de Zariski, luego definiremos el ideal de un conjunto algebraico y daremos algunas propiedades. Definiremos un espacio topológico irreducible y luego daremos una caracterización de dicho espacio topológico, también daremos una caracterización de un conjunto algebraico irreducible y conseguiremos el Corolario 2.1 que será herramienta importante en la prueba central.

Definiremos lo que es una variadad algebraica, función polinomial y luego veremos lo que es una aplicación polinomial y daremos algunos ejemplos que serán importantes para el resultado central.

\footnotetext{
* Programa de Estudios Generales, Universidad de Lima. Lima, Perú. (camejia@ulima.edu.pe).

${ }^{\dagger}$ Programa de Estudios Generales, Universidad de Lima. Lima, Perú.(inunez aulima . edu.pe).

${ }_{\ddagger}^{\ddagger}$ Facultad de Ciencias Matemáticas , Universidad Nacional Mayor de San Marcos. Lima, Perú. (rgalvezp@unmsm.edu.pe).

$\S$ Facultad de Ciencias y Filosofía, Universidad Peruana Cayetano Heredia. Lima, Perú. (neisser.pino@upch.pe).
} 
Por último veremos algunos resultados, el primero dice lo siguiente: si el polinomio característico de la matriz $A \in M_{n \times n}(\mathbb{K})$ es $P_{A}(x)$ entonces existen polinomios $P_{0}, P_{1}, \ldots, P_{n-1} \in \mathbb{K}\left[X_{i j}\right]$ con $i, j=$ $1,2, \ldots, n$ tales que $P_{A}(x)=x^{n}+P_{n-1}(A) x^{n-1}+\ldots+P_{0}(A)$. El segundo resultado afirma que el conjunto $\mathcal{R}=\left\{A \in \mathbf{A}_{\mathbb{K}}^{n^{2}} \mid P_{A}(A)=\operatorname{det}\left(A I_{n}-A\right)=0\right\}$ es un cerrado en la topología de Zariski y en su prueba utilizaremos el primer resultado. El tercer resultado dice que el conjunto

$$
\mathcal{U}:=\left\{A \in \mathbf{A}_{\mathbb{K}}^{n^{2}} \mid \text { A tiene } n \text { autovalores distinos }\right\} .
$$

es un abierto no vacío de la topología de Zariski con $\mathcal{U} \subseteq \mathcal{R}$ donde $\mathbb{K}$ es un cuerpo algebraicamente cerrado. En los resultados anteriores vamos a considerar la clausura algebraica $\overline{\mathbb{K}}$ en vez $\mathbb{K}$ y así el teorema central quedará demostrado.

2. Preliminares. En este trabajo $\mathbb{K}$ denota un cuerpo y $\overline{\mathbb{K}}$ su clausura algebraica.

Definición 2.1. Sea $n \in \mathbb{Z}$ tal que $n \geq 1$. El espacio afín de dimensión $\boldsymbol{n}$ sobre el cuerpo $\mathbb{K}$ se define como

$$
\mathbf{A}_{\mathbb{K}}^{n}:=\left\{\left(a_{1}, a_{2}, \ldots, a_{n}\right) \mid a_{i} \in \mathbb{K}, 1 \leq i \leq n\right\}
$$

Definición 2.2. Un cero o raíz de un polinomio $q\left(x_{1}, x_{2}, \ldots, x_{n}\right) \in \mathbb{K}\left[x_{1}, x_{2}, \ldots, x_{n}\right]$ es un elemento $a=\left(a_{1}, a_{2}, \ldots, a_{n}\right) \in \mathbf{A}_{\mathbb{K}}^{n}$ tal que $q(a)=0$.

Una hipersuperficie es el conjunto de todos los ceros de un polinomio no constante $q \in \mathbb{K}\left[x_{1}, x_{2}, \ldots, x_{n}\right]$, se denota por $\mathcal{Z}(q):=\left\{a \in \mathbf{A}_{\mathbb{K}}^{n} \mid q(a)=0\right\}$. Para más detalles ver [4], página 4.

Definición 2.3. Un conjunto $T \subseteq \mathbf{A}_{\mathbb{K}}^{n}$ es algebraico si $T=\mathcal{Z}(S)$ para algún $S \subseteq \mathbb{K}\left[x_{1}, x_{2}, \ldots, x_{n}\right]$ donde $\mathcal{Z}(S):=\left\{a=\left(a_{1}, a_{2}, \ldots, a_{n}\right) \in \mathbf{A}_{\mathbb{K}}^{n} \mid q(a)=0\right.$, para todo $\left.q\left(x_{1}, x_{2}, \ldots, x_{n}\right) \in S\right\}$.

Observaciones.

1. Si $S=\{q\} \subseteq \mathbb{K}\left[x_{1}, x_{2}, \ldots, x_{n}\right]$, entonces $\mathcal{Z}(\{q\})$ coincide con $\mathcal{Z}(q)$.

2. $\mathcal{Z}(S)=\bigcap_{q \in S} \mathcal{Z}(q)$.

3. Sea $I$ un subconjunto de $\mathbb{K}\left[x_{1}, x_{2}, \ldots, x_{n}\right]$ generado por $S$ entonces $\mathcal{Z}(S)=\mathcal{Z}(I)$.

Esto último quiere decir, que podemos definir sin pérdida de generalidad un conjunto algebraico $U=$ $\mathcal{Z}(I)$ con $I$ ideal de $\mathbb{K}\left[x_{1}, x_{2}, \ldots, x_{n}\right]$ y esto es lo que haremos de ahora en adelante, a menos que se diga lo contrario.

Proposición 2.1. Se cumplen:

1. Sean $I, J$ ideales de $\mathbb{K}\left[x_{1}, x_{2}, \ldots, x_{n}\right]$, si $I \subseteq J$ entonces $\mathcal{Z}(J) \subseteq \mathcal{Z}(I)$.

2. $\mathcal{Z}(0)=\mathbf{A}_{\mathbb{K}}^{n}$ y $\mathcal{Z}(1)=\emptyset$; es decir, los conjuntos $\mathbf{A}_{\mathbb{K}}^{n}, \emptyset$ son algebraicos.

3. Sea $\left\{I_{\lambda}\right\}_{\lambda \in \Lambda}$ una familia de ideales de $\mathbb{K}\left[x_{1}, x_{2}, \ldots, x_{n}\right]$ entonces $\bigcap_{\lambda \in \Lambda} \mathcal{Z}\left(I_{\lambda}\right)=\mathcal{Z}\left(\bigcup_{\lambda \in \Lambda} I_{\lambda}\right)$.

4. Sean $I, J$ ideales de $\mathbb{K}\left[x_{1}, x_{2}, \ldots, x_{n}\right]$ entonces $\mathcal{Z}(I) \cup \mathcal{Z}(J)=\mathcal{Z}(I J)$.

Ver [2], página 56 y [5], página 10.

\subsection{La topología de Zariski.}

Si $\mathcal{F}:=\left\{U \subseteq \mathbf{A}_{\mathbb{K}}^{n} \mid \mathbf{A}_{\mathbb{K}}^{n} \backslash U\right.$ es un conjunto algebraico $\}$ entonces $\left(\mathbf{A}_{\mathbb{K}}^{n}, \mathcal{F}\right)$ es un espacio topológico, pues por la Proposición 2.1 tenemos que $\mathcal{Z}(0)=\mathbf{A}_{\mathbb{K}}^{n}, \mathcal{Z}(1)=\emptyset$, si $\left\{I_{\lambda}\right\}_{\lambda \in \Lambda}$ una familia de ideales de $\mathbb{K}\left[x_{1}, x_{2}, \ldots, x_{n}\right]$ entonces $\bigcap_{\lambda \in \Lambda} \mathcal{Z}\left(I_{\lambda}\right)=\mathcal{Z}\left(\bigcup_{\lambda \in \Lambda} I_{\lambda}\right)$ y $\mathcal{Z}(I) \cup \mathcal{Z}(J)=\mathcal{Z}(I J)$. La topología $\mathcal{F}$ es llamada la topología de Zariski y los $\mathcal{Z}(I)$ son los cerrados de dicha topología.

Ejemplo 2.1. El conjunto $A=\left\{\left(a_{1}, a_{2}\right) \in \mathbf{A}_{\mathbb{K}}^{2} \mid a_{1}=a_{2} \quad o \quad a_{2}=a_{1}^{2}\right\}$ es algebraico.

Ejemplo 2.2. El conjunto $X=\left\{(a, b) \in \mathbf{A}_{\mathbb{R}}^{2} \mid \operatorname{sen}(a)=b\right\}$ no es algebraico.

Para el siguiente ejemplo, vamos a denotar al anillo de polinomios de $n^{2}$ variables como $\mathbb{K}\left[x_{i j}\right]$, luego $p \in \mathbb{K}\left[x_{i j}\right]$ es de la forma $p\left(x_{i j}\right)=p\left(x_{11}, x_{12}, \ldots, x_{1 n}, x_{21}, x_{22}, \ldots, x_{2 n}, \ldots, x_{n 1}, x_{n 2}, \ldots, x_{n n}\right)$.

Ejemplo 2.3. El conjunto $S L_{n}(\mathbb{K}):=\left\{A \in M_{n \times n}(\mathbb{K}) \mid \operatorname{det}(A)=1\right\}$ es algebraico. Basta considerar la biyección natural entre $M_{n \times n}(\mathbb{K})$ y $\mathbf{A}_{\mathbb{K}}^{n^{2}}$ y tomar el polinomio $p\left(x_{i j}\right)=\operatorname{det}\left(x_{i j}\right)-1 \in \mathbb{K}\left[x_{i j}\right]$ donde $\operatorname{det}\left(x_{i j}\right)$ es el determinante de la matriz $\left(x_{i j}\right)_{n \times n}$, luego $S L_{n}(\mathbb{K})=\mathcal{Z}\left(p\left(x_{i j}\right)\right)$.

Ver [2], página 55 para más ejemplos.

Definición 2.4. Sea $X \subseteq \mathbf{A}_{\mathbb{K}}^{n}$. Definimos el ideal del conjunto $X$ como

$$
\mathcal{I}(X):=\left\{p \in \mathbb{K}\left[x_{1}, x_{2}, \ldots, x_{n}\right] \mid p(a)=0, \text { para todo } a \in X\right\}
$$


Proposición 2.2. Se cumplen:

1. $\mathcal{I}(X)$ es un ideal de $\mathbb{K}\left[x_{1}, x_{2}, \ldots, x_{n}\right]$.

2. Si $X \subseteq Y \subseteq \mathbf{A}_{\mathbb{K}}^{n}$ entonces $\mathcal{I}(Y) \subseteq \mathcal{I}(X)$.

3. $\mathcal{I}(\emptyset)=\mathbb{K}\left[x_{1}, x_{2}, \ldots, x_{n}\right]$.

4. $\mathcal{I}\left(\mathbf{A}_{\mathbb{K}}^{n}\right)=\{0\}$ sí y sólo si $\mathbb{K}$ es infinito.

5. $I \subseteq \mathcal{I}(\mathcal{Z}(I))$.

6. $\mathcal{Z}(\mathcal{I}(\mathcal{Z}(I)))=\mathcal{Z}(I)$.

Ver [7] página 3 y [5] página 12.

Definición 2.5. Sea $(X, \mathcal{G})$ un espacio topológico. Decimos que $X$ es reducible si existen $X_{1}$ y $X_{2}$ conjuntos cerrados propios de $X$ tales que $X=X_{1} \cup X_{2}$. En caso contrario decimos que X es irreducible.

Observación 2.1. En la Definición 2.5 si $X=\mathbf{A}_{\mathbb{K}}^{n}$ y $\mathcal{G}$ es la topología de Zariski, tenemos que un conjunto algebrico $\mathcal{Z}$ es reducible si existen conjuntos algebraicos propios $\mathcal{Z}_{1}, \mathcal{Z}_{2}$ de $\mathcal{Z}$ tales que $\mathcal{Z}=$ $\mathcal{Z}_{1} \cup \mathcal{Z}_{2}$.

Ejemplo 2.4. El conjunto algebraico $\mathcal{Z}\left(y^{2}-x y-x^{2} y+x^{3}\right) \subseteq \mathbf{A}_{\mathbb{R}}^{2}$ es reducible pues $\mathcal{Z}\left(y^{2}-x y-\right.$ $\left.x^{2} y+x^{3}\right)=\mathcal{Z}(y-x) \cup \mathcal{Z}\left(y-x^{2}\right)$, con $\mathcal{Z}(y-x), \mathcal{Z}\left(y-x^{2}\right) \subseteq \mathbf{A}_{\mathbb{R}}^{2}$ conjuntos algebraicos propios de $\mathcal{Z}\left(y^{2}-x y-x^{2} y+x^{3}\right)$.

Proposición 2.3. Sea $(X, \mathcal{G})$ un espacio topológico. $X$ es irreducible si y sólo si todo conjunto abierto no vacio $U \subseteq X$ es denso, por tanto $\bar{U}=X$.

Ver [1] página 7.

Ahora veamos una caracterización de los conjuntos algebraicos irreducibles.

Proposición 2.4. Sea $\mathcal{Z}=\mathcal{Z}(I) \subseteq \mathbf{A}_{\mathbb{K}}^{n}$ un conjunto algebraico. $\mathcal{Z}$ es irreducible sí y sólo si $\mathcal{I}(\mathcal{Z}) \subseteq$ $\mathbb{K}\left[x_{1}, x_{2}, \ldots, x_{n}\right]$ es un ideal primo.

Demostración:

$[\Rightarrow] \quad \mathrm{Si} \mathcal{I}(\mathcal{Z})$ no es un ideal primo existen $p, q \in \mathbb{K}\left[x_{1}, x_{2}, \ldots, x_{n}\right]$ tales que $p \cdot q \in \mathcal{I}(\mathcal{Z})$ pero $p \notin \mathcal{I}(\mathcal{Z})$ y $q \notin \mathcal{I}(\mathcal{Z})$. Dado que $p \cdot q \in \mathcal{I}(\mathcal{Z})$ entonces $\{p \cdot q\} \subseteq \mathcal{I}(\mathcal{Z})$ luego $\mathcal{Z}(\{p \cdot q\}) \supseteq \mathcal{Z}(\mathcal{I}(\mathcal{Z}))=\mathcal{Z}$.

Tenemos

$$
\mathcal{Z}=\mathcal{Z} \cap \mathcal{Z}(p \cdot q)=\mathcal{Z} \cap[\mathcal{Z}(p) \cup \mathcal{Z}(q)]=[\mathcal{Z} \cap \mathcal{Z}(p)] \cup[\mathcal{Z} \cap \mathcal{Z}(q)]
$$

Como $p \notin \mathcal{I}(\mathcal{Z})$ entonces $p(a) \neq 0$, para algún $a \in \mathcal{Z}$, es decir $a \notin \mathcal{Z}(p)$ luego $\mathcal{Z}(p)$ es un subconjunto propio de $\mathcal{Z}$ entonces $\mathcal{Z} \cap \mathcal{Z}(p)$ es un subconjunto propio de $\mathcal{Z}$. De forma similar $\mathcal{Z} \cap \mathcal{Z}(q)$ es un subconjunto propio de $\mathcal{Z}$. Por tanto $\mathcal{Z}$ es reducible.

$[\Leftarrow]$ Supongamos que $\mathcal{Z}$ es reducible entonces existen $\mathcal{Z}_{1}=\mathcal{Z}_{1}\left(I_{1}\right), \mathcal{Z}_{2}=\mathcal{Z}_{2}\left(I_{2}\right) \subseteq \mathbf{A}_{\mathbb{K}}^{n}$ conjuntos algebraicos propios de $\mathcal{Z}$ tales que $\mathcal{Z}=\mathcal{Z}_{1} \cup \mathcal{Z}_{2}$, luego $\mathcal{Z}_{1} \subseteq \mathcal{Z}$ y $\mathcal{Z}_{2} \subseteq \mathcal{Z}$ entonces $\mathcal{I}\left(\mathcal{Z}_{1}\right) \supseteq \mathcal{I}(\mathcal{Z})$ e $\mathcal{I}\left(\mathcal{Z}_{2}\right) \supseteq \mathcal{I}(\mathcal{Z})$. Es fácil ver que existen $f_{1} \in \mathcal{I}\left(\mathcal{Z}_{1}\right), f_{2} \in \mathcal{I}\left(\mathcal{Z}_{2}\right)$ tales que $f_{1}, f_{2} \notin \mathcal{I}(\mathcal{Z})$.

Ahora si $a \in \mathcal{Z}$ entonces $a \in \mathcal{Z}_{1}$ ó $a \in \mathcal{Z}_{2}$. Tenemos dos casos:

Si $a \in \mathcal{Z}_{1}$ entonces $f_{1}(a)=0$ luego $\left(f_{1} \cdot f_{2}\right)(a)=0$ esto quiere decir que $f_{1} \cdot f_{2} \in \mathcal{I}(\mathcal{Z})$.

Si $a \in \mathcal{Z}_{2}$ entonces $f_{2}(a)=0$ luego $\left(f_{1} \cdot f_{2}\right)(a)=0$ esto quiere decir que $f_{1} \cdot f_{2} \in \mathcal{I}(\mathcal{Z})$.

Por tanto $\mathcal{I}(\mathcal{Z})$ no es ideal primo.

Corolario 2.1. Si $\mathbb{K}$ es infinito entonces $\mathbf{A}_{\mathbb{K}}^{n}$ es irreducible.

Demostración: Como $\mathbb{K}$ es infinito entonces $\mathcal{I}\left(\mathbf{A}_{\mathbb{K}}^{n}\right)=\{0\}$ y dado que $\{0\} \subseteq \mathbb{K}\left[x_{1}, x_{2}, \ldots, x_{n}\right]$ es ideal primo entonces por Proposición $2.4 \mathbf{A}_{\mathbb{K}}^{n}$ es irreducible.

Definición 2.6. Una variedad algebraica de $\mathbf{A}_{\mathbb{K}}^{n}$ es un conjunto algebraico irreducible de $\mathbf{A}_{\mathbb{K}}^{n}$.

Definición 2.7. Sea $\mathcal{Z}$ una variedad algebraica no vacía de $\mathbf{A}_{\mathbb{K}}^{n}$. La función $F: \mathcal{Z} \longrightarrow \mathbb{K}$ es llamada una función polinomial sobre $\mathcal{Z}$ si existe un polinomio $p \in \mathbb{K}\left[x_{1}, x_{2}, \ldots, x_{n}\right]$ tal que $F\left(a_{1}, a_{2}, \ldots, a_{n}\right)=$ $p\left(a_{1}, a_{2}, \ldots, a_{n}\right)$ para cada $\left(a_{1}, a_{2}, \ldots, a_{n}\right) \in \mathcal{Z}$.

Definición 2.8. Sean $\mathcal{Z}_{1} \subseteq \mathbf{A}_{\mathbb{K}}^{n} y \mathcal{Z}_{2} \subseteq \mathbf{A}_{\mathbb{K}}^{m}$ variedades algebraicas no vacías. Una función $\varphi: \mathcal{Z}_{1} \longrightarrow$ $\mathcal{Z}_{2}$ es llamada aplicación polinómica si existen polinomios $p_{1}, p_{2}, \ldots, p_{m} \in \mathbb{K}\left[x_{1}, x_{2}, \ldots, x_{n}\right]$ tales que $\varphi\left(a_{1}, a_{2}, \ldots, a_{n}\right)=\left(p_{1}\left(a_{1}, a_{2}, \ldots, a_{n}\right), p_{2}\left(a_{1}, a_{2}, \ldots, a_{n}\right), \ldots, p_{m}\left(a_{1}, a_{2}, \ldots, a_{n}\right)\right)$ para cada $\left(a_{1}, a_{2}, \ldots, a_{n}\right) \in$ $\mathcal{Z}_{1}$.

Para el siguiente ejemplo vamos a considerar que una matriz $A=\left(a_{i j}\right)_{n \times n} \in M_{n \times n}(\mathbb{K})$ puede ser vista como un elemento de $\mathbf{A}_{\mathbb{K}}^{n^{2}}$ entonces $A=\left(a_{11}, a_{12}, \ldots, \ldots, a_{n n}\right)$.

Ejemplo 2.5. La función $\varphi_{1}: \mathbf{A}_{\mathbb{K}}^{n^{2}} \longrightarrow \mathbf{A}_{\mathbb{K}}^{n^{2}}, \varphi_{1}(A):=A=\left(a_{i j}\right)_{n \times n}$ es una aplicación polinómica pues existen polinomios $p_{11}\left(x_{i j}\right)=x_{11}, p_{12}\left(x_{i j}\right)=x_{12}, \ldots, p_{n n}\left(x_{i j}\right)=x_{n n}$ en $\mathbb{K}\left[x_{i j}\right]$ con $i, j=$ $1,2, \ldots, n$ tales que $\varphi\left(a_{11}, a_{12}, \ldots, a_{n n}\right)=\varphi(A)=A=\left(a_{11}, a_{12}, \ldots, a_{n n}\right)=\left(p_{11}\left(a_{i j}\right), p_{12}\left(a_{i j}\right), \ldots, p_{n n}\left(a_{i j}\right)\right)$. 
De forma similar se prueba que las funciones $\varphi_{i}: \mathbf{A}_{\mathbb{K}}^{n^{2}} \longrightarrow \mathbf{A}_{\mathbb{K}}^{n^{2}}, \varphi_{i}(A):=A^{i}$ son aplicaciones polinómicas para $i=2,3, \ldots, n$.

3. Resultado central. Antes de demostrar el teorema de Cayley-Hamilton, veremos algunos resultados.

Lema 3.1. Sea $P_{A}(x):=\operatorname{det}\left(x I_{n}-A\right)$ el polinomio característico de la matriz $A \in M_{n \times n}(\mathbb{K}) \cong$ $\mathbf{A}_{\mathbb{K}}^{n^{2}}$. Para cada $A \in M_{n \times n}(\mathbb{K})$ existen $P_{0}, P_{1}, \ldots, P_{n-1} \in \mathbb{K}\left[x_{i j}\right]$ con $i, j=1,2, \ldots, n$ tales que $P_{A}(x)=$ $x^{n}+P_{n-1}(A) x^{n-1}+\ldots+P_{1}(A) x+P_{0}(A)$.

Demostración: Inducción sobre $\mathrm{n}$.

Para $n=2$. Si $A=\left(\begin{array}{ll}a & b \\ c & d\end{array}\right)$ entonces $P_{A}(x)=x^{2}+(-a-d) x+(a d-b c)$, luego existen polinomios $P_{1}\left(x_{1}, x_{2} . x_{3}, x_{4}\right)=-x_{1}-x_{4}, P_{0}\left(x_{1}, x_{2} . x_{3}, x_{4}\right)=x_{1} x_{4}-x_{2} x_{3}$ en $\mathbb{K}\left[x_{1}, x_{2}, x_{3}, x_{4}\right]$ tales que $P_{A}(x)=$ $x^{2}+P_{1}(A) x+P_{0}(A)$.

Dada $A=\left(a_{i j}\right)_{n \times n} \in M_{n \times n}(\mathbb{K})$ y sin pérdida de generalidad supongamos que $n$ es impar.

Tenemos:

$$
\begin{gathered}
P_{A}(x)=\operatorname{det}\left(\begin{array}{cccc}
x-a_{11} & -a_{12} & \cdots & -a_{1 n} \\
-a_{21} & x-a_{22} & \cdots & -a_{2 n} \\
\vdots & \vdots & \ddots & \vdots \\
-a_{n 1} & -a_{n 2} & \cdots & x-a_{n n}
\end{array}\right) \\
=\operatorname{det}\left(E_{n 1}\right)\left(-a_{n 1}\right)-\operatorname{det}\left(E_{n 2}\right)\left(-a_{n 2}\right)+\ldots-\operatorname{det}\left(E_{n(n-1)}\right)\left(-a_{n(n-1)}\right) \\
+\operatorname{det}\left(\begin{array}{cccc}
x-a_{11} & -a_{12} & \cdots & -a_{1(n-1)} \\
-a_{21} & x-a_{22} & \cdots & -a_{2(n-1)} \\
\vdots & \vdots & \ddots & \vdots \\
-a_{(n-1) 1} & -a_{(n-1) 2} & \cdots & x-a_{(n-1)(n-1)}
\end{array}\right)\left(x-a_{n n}\right),
\end{gathered}
$$

donde $E_{n i}$ es una submatriz de la matriz $x I_{n}-A$ obtenida de eliminar la $n$-sima fila y la $i$-ésima columna para cada $i=1,2, \ldots, n-1$.

El determinante que está multiplicando a $\left(x-a_{n n}\right)$ es el polinomio característico de alguna matriz $B \in M_{(n-1)(n-1)}(\mathbb{K})$; es decir,

$$
P_{B}(x)=\operatorname{det}\left(\begin{array}{cccc}
x-a_{11} & -a_{12} & \cdots & -a_{1(n-1)} \\
-a_{21} & x-a_{22} & \cdots & -a_{2(n-1)} \\
\vdots & \vdots & \ddots & \vdots \\
-a_{(n-1) 1} & -a_{(n-1) 2} & \cdots & x-a_{(n-1)(n-1)}
\end{array}\right) \text {. }
$$

Luego, por la hipótesis inductiva existen $P_{0}, P_{1}, \ldots, P_{n-2} \in \mathbb{K}\left[x_{i j}\right]$ para cada $i, j=1,2, \ldots, n-1$ tales que

$$
P_{B}(x)=x^{n-1}+P_{n-2}(B) x^{n-2}+\ldots+P_{1}(B) x+P_{0}(B) .
$$

También observemos que $\operatorname{det}\left(E_{n 1}\right), \operatorname{det}\left(E_{n 2}\right), \ldots, \operatorname{det}\left(E_{n(n-1)}\right)$ son polinomios de grado $n-2$.

Tenemos:

$$
\begin{aligned}
& \operatorname{det}\left(E_{n 1}\right)=r_{1} x^{n-2}+b_{n-3}^{(1)} x^{n-3}+\ldots+b_{1}^{(1)} x+b_{0}^{(1)}, \\
& \operatorname{det}\left(E_{n 2}\right)=r_{2} x^{n-2}+b_{n-3}^{(2)} x^{n-3}+\ldots+b_{1}^{(2)} x+b_{0}^{(2)},
\end{aligned}
$$


Sea

$$
\operatorname{det}\left(E_{n(n-1)}\right)=r_{n-1} x^{n-2}+b_{n-3}^{(n-1)} x^{n-3}+\ldots+b_{1}^{(n-1)} x+b_{0}^{(n-1)} .
$$

$$
\begin{aligned}
L & =-a_{n 1} \operatorname{det}\left(E_{n 1}\right)+a_{n 2} \operatorname{det}\left(E_{n 2}\right)-\ldots+a_{n(n-1)} \operatorname{det}\left(E_{n(n-1)}\right) \\
& =\left[-r_{1} a_{n 1}+r_{2} a_{n 2}-\ldots+r_{n-1} a_{n(n-1)}\right] x^{n-2}+ \\
& +\left[-a_{n 1} b_{n-2}^{(1)}+a_{n 2} b_{n-2}^{(2)}-\ldots+a_{n(n-1)} b_{n-2}^{(n-1)}\right] x^{n-3}+\ldots \\
& +\ldots+\left[-a_{n 1} b_{1}^{(1)}+a_{n 2} b_{1}^{(2)}-\ldots+a_{n(n-1)} b_{1}^{(n-1)}\right] x+ \\
& +\left[-a_{n 1} b_{0}^{(1)}+a_{n 2} b_{0}^{(2)}-\ldots+a_{n(n-1)} b_{0}^{(n-1)}\right] .
\end{aligned}
$$

Tenemos

$$
\begin{aligned}
P_{A}(x) & =-a_{n 1} \operatorname{det}\left(E_{n 1}\right)+a_{n 2} \operatorname{det}\left(E_{n 2}\right)-\ldots+a_{n(n-1)} \operatorname{det}\left(E_{n(n-1)}\right)+P_{B}(x)\left(x-a_{n n}\right) \\
& =L+\left[x^{n-1}+P_{n-2}(B) x^{n-2}+P_{n-3}(B) x^{n-3}+\ldots+P_{1}(B) x+P_{0}(B)\right]\left(x-a_{n n}\right) \\
& =L+x^{n}+\left(P_{n-2}(B)-a_{n n}\right) x^{n-1}+\left(P_{n-3}(B)-a_{n n} P_{n-2}(B)\right) x^{n-2}+\ldots \\
& +\left(P_{0}(B)-a_{n n} P_{1}(B)\right) x-a_{n n} P_{0}(B) \\
& =x^{n}+\left[P_{n-2}(B)-a_{n n}\right] x^{n-1}+\ldots \\
& +\left[P_{n-3}(B)-r_{1} a_{n 1}+r_{2} a_{n 2}-\ldots+r_{n-1} a_{n(n-1)}-P_{n-2}(B) a_{n n}\right] x^{n-2} \\
& +\ldots+\left[P_{0}(B)-a_{n 1} b_{1}^{(1)}+a_{n 2} b_{1}^{(2)}-\ldots+a_{n(n-1)} b_{1}^{(n-1)}-a_{n n} P_{1}(B)\right] x \\
& -a_{n 1} b_{0}^{(1)}+a_{n 2} b_{0}^{(2)}-\ldots+a_{n(n-1)} b_{0}^{(n-1)}-a_{n n} P_{0}(B) .
\end{aligned}
$$

Por tanto existen $\quad \widetilde{P}_{0}\left(x_{i j}\right), \widetilde{P}_{1}\left(x_{i j}\right), \ldots, \widetilde{P}_{n-1}\left(x_{i j}\right) \in \mathbb{K}\left[x_{i j}\right] \quad$ con $i, j=1,2, \ldots, n$ tales que $P_{A}(x)=x^{n}+\widetilde{P}_{n-1}(A) x^{n-1}+\ldots+\widetilde{P}_{1}(A) x+\widetilde{P}_{0}(A)$.

Lema 3.2. El conjunto $\mathcal{R}=\left\{A \in M_{n \times n}(\mathbb{K}) \mid P_{A}(A):=\operatorname{det}\left(A I_{n}-A\right)=0\right\}$ es un cerrado en la topología de Zariski.

Demostración: Por Lema 3.1, para cada $A \in M_{n \times n}(\mathbb{K})$ existen $P_{0}, P_{1}, \ldots, P_{n-1} \in \mathbb{K}\left[x_{i j}\right]$ con $i, j=1,2, \ldots, n$ tales que $P_{A}(x)=x^{n}+P_{n-1}(A) x^{n-1}+\ldots+P_{1}(A) x+P_{0}(A)$.

Sabemos que las funciones $\varphi_{i}: \mathbf{A}_{\mathbb{K}}^{n^{2}} \longrightarrow \mathbf{A}_{\mathbb{K}}^{n^{2}}, \varphi_{i}(A):=A^{i}$ son aplicaciones polinómicas para cada $i=1,2, \ldots, n$. Ahora definimos la función $\Psi: \mathbf{A}_{\mathbb{K}}^{n^{2}} \longrightarrow \mathbf{A}_{\mathbb{K}}^{n^{2}}$,

$$
\Psi(A):=P_{0}(A)+P_{1}(A) \varphi_{1}(A)+P_{n-1}(A) \varphi_{n-1}(A)+\varphi_{n}(A)=P_{A}(A) .
$$

Es fácil ver que $\Psi$ es una aplicación polinómica, luego existen $\Psi_{11}\left(x_{i j}\right), \Psi_{12}\left(x_{i j}\right), \ldots, \Psi_{n n}\left(x_{i j}\right) \in \mathbb{K}\left[x_{i j}\right]$ para cada $i, j=1,2, \ldots, n$ tales que

$$
\Psi(A)=\left(\Psi_{11}(A), \Psi_{12}(A), \ldots, \Psi_{n n}(A)\right) .
$$

Afirmación: Sea $\mathcal{I}=\left\langle\Psi_{11}, \Psi_{12}, \ldots, \Psi_{n n}\right\rangle$ un ideal de $\mathbb{K}\left[x_{i j}\right]$ para cada $i, j=1,2, \ldots, n$. Se cumple que $\mathcal{R}=\mathcal{Z}(\mathcal{I})$. En efecto, $A \in \mathcal{R}$ si y sólo si $0=P_{A}(A)=\Psi(A)=\left(\Psi_{11}(A), \Psi_{12}(A), \ldots, \Psi_{n n}(A)\right)$ si y sólo si $\Psi_{11}(A)=\Psi_{12}(A)=\ldots=\Psi_{n n}(A)=0$ si y sólo si $Q(A)=0$ para cada $Q \in \mathcal{I}$ si y solo si $A \in \mathcal{Z}(\mathcal{I})$. Zariski.

De la afirmación tenemos que $\mathcal{R}$ es un conjunto algebraico y por tanto es un cerrado en la topología de

Ahora veremos algunos resultados del Álgebra Lineal en una proposición y algunos resultados sobre el discriminante de polinomios.

Proposición 3.1. Sea A una matriz en $M_{n \times n}(\mathbb{K})$. Entonces se cumplen:

1. Si A tiene $n$ autovalores diferentes entonces sus autovectores asociados son linealmente independientes.

2. Si $B \in M_{n \times n}(\mathbb{K})$ es semejante a la matriz A entonces sus polinomios característicos son iguales.

3. A es diagonalizable si y solo si tiene $n$ autovectores linealmente independientes. En tal caso la matriz diagonal $D$ semejante a la matriz A está dada por 


$$
D=\left(\begin{array}{cccc}
\lambda_{1} & 0 & \cdots & 0 \\
0 & \lambda_{2} & \cdots & 0 \\
\vdots & \vdots & \ddots & \vdots \\
0 & 0 & \cdots & \lambda_{n}
\end{array}\right)
$$

donde los $\lambda_{i}$ con $i=1,2, \ldots, n$ son los autovalores de $A$.

Ver [9], páginas 555 hasta 559.

Definición 3.1. Sea $P(x)=a_{n}\left(x-r_{1}\right)\left(x-r_{2}\right) \ldots\left(x-r_{n}\right)$ un polinomio de grado $n \geq 1$ con coeficientes en $\mathbb{K}$ y no necesariamente raices distintas $r_{1}, r_{2}, \ldots, r_{n}$ en $\overline{\mathbb{K}}$. Entonces el discriminante de $P(x)$ es

$$
\operatorname{Disc}(P(x)):=a_{n}^{2 n-2} \prod_{1 \leq i<j \leq n}\left(r_{i}-r_{j}\right)^{2} \in \mathbb{K} .
$$

Ver [6], página 179.

Observación 3.1. Disc $(P(x)) \neq 0$ si y sólo si las raices $r_{1}, r_{2}, \ldots, r_{n}$ en $\overline{\mathbb{K}}$ son distintas.

Lema 3.3. Sea $\mathbb{K}$ un cuerpo algebraicamente cerrado y consideremos el conjunto

$$
\mathcal{U}:=\left\{A \in M_{n \times n}(\mathbf{K}) \mid A \text { tiene } n \text { autovalores distinos }\right\} .
$$

Entonces $\mathcal{U}$ es un abierto no vacío de la topología de Zariski y $\mathcal{U} \subseteq \mathcal{R}$.

Demostración: Primero veamos que $\mathcal{U} \subseteq \mathcal{R}$. En efecto, dado $A \in \mathcal{U}$ entonces A tiene $\mathrm{n}$ autovalores distintos a los cuales denotaremos por $\lambda_{1}, \lambda_{2}, \ldots \lambda_{n} \in \mathbb{K}$, luego A tiene $\mathrm{n}$ autovectores $v_{1}, v_{2}, \ldots, v_{n}$ linealmente independientes asociados a $\operatorname{los} \lambda_{i} \operatorname{con} i=1,2, \ldots, n$. $\left(A v_{i}=\lambda_{i} v_{i}\right.$ para cada $\left.i=1,2, \ldots, n\right)$.

Tenemos que A es diagonalizable, es decir A es semejante a una matriz diagonal de la forma

$$
D=\left(\begin{array}{cccc}
\lambda_{1} & 0 & \cdots & 0 \\
0 & \lambda_{2} & \cdots & 0 \\
\vdots & \vdots & \ddots & \vdots \\
0 & 0 & \cdots & \lambda_{n}
\end{array}\right)
$$

entonces los polinomios característicos de las matrices A y D son iguales, es decir $P_{A}(x)=P_{D}(x)=$ $\left(x-\lambda_{1}\right)\left(x-\lambda_{2}\right) \cdots\left(x-\lambda_{n}\right)$. Es fácil ver que $P_{A}(A) v_{i}=0$ para cada $i=1,2, \ldots, n$ y dado que $\beta=\left\{v_{1}, v_{2}, \ldots, v_{n}\right\}$ es una $\mathbb{K}$-base de $\mathbf{A}_{\mathbb{K}}^{n}$ entonces $P_{A}(A)=0$. Por tanto $A \in \mathcal{R}$.

Ahora veamos que $\mathcal{U}$ es un abierto en la topología de Zariski. En efecto, definimos la función

$$
F: \mathbf{A}_{\mathbb{K}}^{n^{2}} \longrightarrow \mathbb{K}, \quad F(A):=\operatorname{Disc}\left(P_{A}(x)\right) .
$$

Es fácil ver que $F$ es una función polinomial es decir existe un polinomio $P \in \mathbb{K}\left[x_{i j}\right] \operatorname{con} i, j=1,2 \ldots, n$ tal que $F(A)=P(A)$, para cada $A \in \mathbf{A}_{\mathbb{K}}^{n^{2}}$.

Tomemos $A \in \mathcal{U}$ entonces el polinomio caraterístico de $A$ es de la forma

$$
P_{A}(x)=\left(x-r_{1}\right)\left(x-r_{2}\right) \cdots\left(x-r_{n}\right),
$$

donde $\operatorname{los} r_{i} \in \mathbb{K}$ con $i=1,2, \ldots, n$ son los autovalores de A, luego

$$
F(A):=\operatorname{Disc}(P(x)):=a_{n}^{2 n-2} \prod_{1 \leq i<j \leq n}\left(r_{i}-r_{j}\right)^{2} \neq 0 .
$$

Por ser $F$ una función polinomial existe un polinomio $p \in \mathbb{K}\left[x_{i j}\right]$ con $i, j=1,2 \ldots, n$ tal que $0 \neq$ $F(A)=p(A)$, si y sólo si $A \notin \mathcal{Z}(p)$. Por tanto $\mathcal{U}=\mathbf{A}_{\mathbb{K}}^{n^{2}}-\mathcal{Z}(p)$.

Ahora ya tenemos las herramientas para probar el teorema central de este trabajo. 
Teorema 3.1 (Teorema de Cayley-Hamilton).

Sean $A \in M_{n \times n}(\mathbb{K}), P_{A}(x) \in \mathbb{K}[x]$ el polinomio característico de A. Entonces $P_{A}(A)=0$.

Demostración: Sabemos que los conjuntos $\mathcal{R}_{1}=\left\{B \in \mathbf{A}_{\mathbb{K}}^{n^{2}} \mid P_{A}(B):=\operatorname{det}\left(B I_{n}-A\right)=0\right\}$ es un cerrado de la topología de Zariski y $\mathcal{U}_{1}:=\left\{A \in \mathbf{A}_{\overline{\mathbb{K}}}^{n^{2}} \mid\right.$ A tiene $n$ autovalores distinos $\} \neq \emptyset$ es abierto con $\mathcal{U}_{1} \subseteq \mathcal{R}_{1}$ por los Lemas 3.2 y 3.3 respectivamente.

Por otro lado $\overline{\mathbb{K}}$ es algebraicamente cerrado, luego es infinito, ver [5], entonces por Corolario $2.1 \mathbf{A}_{\overline{\mathbb{K}}}^{n^{2}}$ es irreducible luego tenemos por Proposición 2.3 que $\overline{\mathcal{U}}_{1}=\mathbf{A}_{\mathbb{K}}^{n^{2}}$.

Ya que $\mathbb{K} \subseteq \overline{\mathbb{K}}$ entonces $\mathbf{A}_{\mathbb{K}}^{n^{2}} \subseteq \mathbf{A}_{\bar{K}}^{n^{2}}=\overline{\mathcal{U}}_{1} \subseteq \overline{\mathcal{R}}_{1}=\mathcal{R}_{1}$ luego $\mathbf{A}_{\mathbb{K}}^{n^{2}} \subseteq \mathcal{R}_{1}$, esto quiere decir que para cada $A \in M_{n \times n}(\mathbb{K})$ se tiene que $A \in \mathcal{R}_{1}$, por tanto $P_{A}(A)=0$.

4. Conclusiones. Este trabajo se desarrolló con la finalidad de desenvolver el artículo [8]. Hemos tratado de explicar con más detalles algunos resultados, como por ejemplo los Lemas 3.1, 3.2 y 3.3. Hemos demostrado algunos resultados de la geometría algebraica, como por ejemplo la Proposición 2.4 y el Corolario 2.1, que son utilizados en la demostración del teorema central. También es importante mencionar que las definiciones y resultados de este trabajo son la base para poder continuar con esta área tan bonita que es la geometría algebraica.

5. Agradecimientos. Se quiere expresar un especial agradecimiento a Aislan Leal, Victor Mielly y a Carlos Sáez Calvo más conocido como "geómetracat"por las observaciones y sugerencias de este trabajo; como también al profesor Tomás Núñez Lay por la revisión de la redacción.

ORCID and License

Carlos Mejía Alemán https:// orcid.org/0000-0002-5081-9175

Irene Edith Núñez Rodriguez https: / / orcid.org/0000-0001-7132-0501

Rodolfo José Gálvez Pérez https://orcid.org/0000-0002-6349-7793

Neisser Pino Romero https://orcid.org/0000-0002-9865-5974

This work is licensed under the Creative Commons - Attribution 4.0 International (CC BY 4.0)

\section{Referencias}

[1] Beshenov A. Invitación a la teoría de esquemas. Apuntes de clase. Universidad de El Salvador, 2019. Recuperado de https: //cadadr.org/san-salvador/2019-esquemas/esquemas.pdf

[2] Borges H, Tengan E. Álgebra comutativa em quatro movimentos. Projeto Euclides, Instituto de Matemática Pura e Aplicada (IMPA), 2015. Recuperado de https://loja.sbm.org.br/index.php/ algebra-comutativa-em-quatro-movimentos.html

[3] Cox D, Little J. O'Shea D. Ideals, Varieties, and Algorithms. An Introduction to Computational Algebraic Geometry and Commutative Algebra. Undergraduate Texts in Mathematics. Springer, 2007. Recuperado de https://doc.lagout.org/science/0_Computer\%20Science/2_Algorithms/Ideals\%2C\%

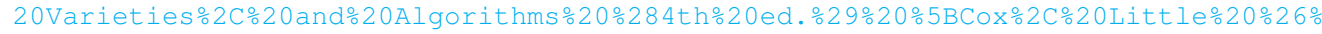
$200 \div 27$ Shea $202015-06-14 \div 5$ D.pdf

[4] Fulton W. Algebraic curves: An introduction to algebraic geometry. University of Michigan, 2008. Recuperado de http: // www. math.lsa.umich.edu/ wfulton/CurveBook.pdf

[5] Gathmann A. Algebraic Geometry-Notes. University of Kaiserslautern, 2003. Recuperado de https: // www . mathematik. uni-kl.de/ gathmann/class/alggeom-2002/alggeom-2002.pdf

[6] Grillet PA. Abstract algebra. Graduate Texts in Mathematics. Editorial Board, Springer, 2000. Recuperado de http: //dobrochan.ru/src/pdf/1204/Grillet_P._A._-_Abstract_Algebra_(2007) (684).pdf

[7] Hartshorne R. Algebraic Geometry. Graduated Texts in Mathematics, Editorial Board, Springer-Verlag, 1977. Recuperado de http://userpage.fu-berlin.de/aconstant/Alg2/Bib/Hartshorne.pdf

[8] Jeffrey A, Rosoff G, College A. A topological proof of the Cayley-Hamilton theorem. Missouri Journal of Mathematical Sciences, 1995, 7(2):63-67. DOI: $10.35834 / 1995 / 0702063$

[9] Stanley I, Grossman S. Álgebra Lineal. University of Montana, University College London. Editorial McGraw-Hill, 2007. Recuperado de http://up-rid2.up.ac.pa:8080/xmlui/bitstream/handle/123456789/1326/\%C3\% 81 lgebra:20lineal. pdf? sequence=1\&isAllowed=y 